\title{
Finite formation time effects for in-medium parton splittings
}

\section{Fabio Domínguez}

Instituto Galego de Física de Altas Enerxías (IGFAE), Universidade de Santiago de Compostela, Santiago de Compostela, 15782 Galicia, Spain

E-mail: fabio.dominguez@usc.es

\section{José Guilherme Milhano}

LIP, Av. Prof. Gama Pinto, 2, P-1649-003 Lisbon, Portugal; Instituto Superior Técnico (IST),

Universidade de Lisboa, Av. Rovisco Pais 1, 1049-001 Lisboa, Portugal

E-mail: gmilhano@lip.pt; guilherme.milhano@tecnico.ulisboa.pt

\section{Carlos A. Salgado}

Instituto Galego de Física de Altas Enerxías (IGFAE), Universidade de Santiago de Compostela, Santiago de Compostela, 15782 Galicia, Spain

E-mail: carlos.salgadocusc.es

\section{Konrad Tywoniuk}

Department of Physics and Technology, University of Bergen, Allegaten 55, 5007 Bergen,

Norway

E-mail: konrad.tywoniuk@uib.no

\section{Víctor Vila*}

Instituto Galego de Física de Altas Enerxías (IGFAE), Universidade de Santiago de Compostela, Santiago de Compostela, 15782 Galicia, Spain

E-mail: victor.vila@usc.es

\begin{abstract}
We present a novel setup of a quark-antiquark antenna splitting inside a color deconfined medium considering the finite formation time of the dipole, which becomes an important scale in the physical picture. In particular, we consider the splitting of a color singlet dipole, studying in detail its formation and subsequent propagation through the medium. We discuss the role of color coherence and the relevant time scales which control the scenario, while also providing theoretical support for vacuum-like emissions early in the medium. Finally, by mapping the spectrum of inmedium splittings through the corresponding kinematical Lund diagram, we elicit regimes of a close correspondence to a semi-classical description and regimes where this description breaks down.
\end{abstract}

International Conference on Hard and Electromagnetic Probes of High-Energy Nuclear Collisions 30 September - 5 October 2018

Aix-Les-Bains, Savoie, France

\footnotetext{
* Speaker.
} 


\section{Introduction}

A total understanding of jet evolution in matter depends to a great extent in being able to fully grasp how the collimated multiparticle sprays called jets behave when these high-energy objects produced from early stage collisions go through a dense medium as the quark-gluon plasma. Not only have they reflected its capacity in probing the QCD parton cascades, but also opening many doors of opportunity to break down the inner structure of these effective tools and constructing new observables to go further in determining its properties. The successes of jet quenching studies in heavy-ion collisions testify to the drawing power of these objects when describing the medium as well as the propagation and fragmentation of jet partons [1].

\section{Results}

\subsection{The physical scenario}

In order to shed new light on the aspects introduced above, in this work we target the problem of a collinear parton splitting in a dense medium by considering the production of a color-singlet final state. In particular, we consider the splitting of photon into a quark-antiquark pair, $\gamma \rightarrow q \bar{q}$, as pictured below in Fig. 1.

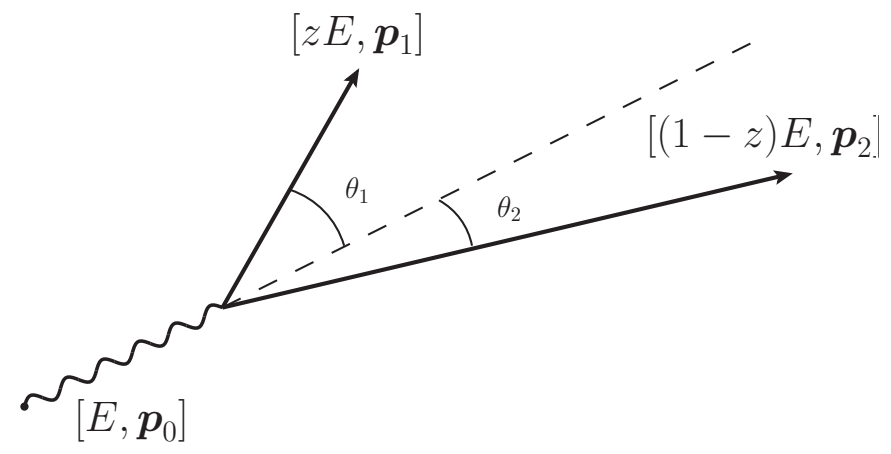

Figure 1: Schematic view of the splitting of a parent parton with momentum $\vec{p}_{0}$ into two daughter partons. We particularize it to produce a color singlet final state, namely $\gamma \rightarrow q \bar{q}$.

In terms of the approaches implemented to face the raised problem, we focus our attention to in-medium hard splittings. In particular, in order to carry out the "semi-classical" framework we have to both impose eikonal trajectories to the particles and fix a shared reference point for the dipole afterwards.

\subsection{Computing the spectrum}

Let us consider a $q \bar{q}$-antenna with short formation time. Taking the medium averages as local in time, the region characterized by the time-like separation between the formation of the dipole in amplitude and complex-conjugate amplitude and the one delimited by the formation time and the length of the medium can be considered independently - hereinafter referred to as Region I and II respectively. 
Region I is analytically described by the two-point function,

$$
S_{i j}\left(t^{\prime}, t\right)=\frac{1}{N_{c}}\left\langle\operatorname{tr} V_{i} V_{j}^{\dagger}\right\rangle
$$

since the Wilson lines build up a dipole which increasingly separates with $t$. In the harmonic approximation, the dipole can be computed as [2]

$$
S_{i j}\left(t^{\prime}, t\right)=\exp \left[-\frac{1}{4} \int_{t}^{t^{\prime}} d s \hat{q} \vec{r}_{i j}^{2}\right],
$$

where $\hat{q}$ is the transport coefficient and $\vec{r}_{i j}$ are the separations traced by the partons.

Region II is more sensitive to medium effects when the partons propagate through it. In particular, we find a four-point function,

$$
Q\left(t^{\prime}, t\right)=\frac{1}{N_{c}}\left\langle\operatorname{tr} V_{1} V_{2}^{\dagger} V_{\overline{2}} V_{\overline{1}}^{\dagger}\right\rangle,
$$

also known as a quadrupole, which turns out to be essential to account for the medium interactions in the final stage of the evolution of the system. It can be easily computed in the Large- $N_{c}$ limit as follows,

$$
Q\left(t^{\prime}, t\right)=S_{1 \overline{1}}\left(t^{\prime}, t\right) S_{2 \overline{2}}\left(t^{\prime}, t\right)+\int_{t}^{t^{\prime}} d s S_{1 \overline{1}}\left(t^{\prime}, s\right) S_{2 \overline{2}}\left(t^{\prime}, s\right) T(s) S_{12}(s, t) S_{\overline{1} \overline{2}}(s, t),
$$

where $T(s)$ is the transition amplitude [3].

In order to find out different regimes when looking at medium interactions, we define the cross section of the process by

$$
\frac{d \sigma^{\text {med }}}{d z d \vec{p}^{2}}=\frac{d \sigma^{v a c}}{d z d \vec{p}^{2}}\left(1+F_{m e d}\right)
$$

where $F_{m e d}$ takes in all the information about the medium modifications,

$$
F_{\text {med }}=2 \int_{0}^{\zeta_{L}} d s\left[\int_{0}^{\zeta_{L}} d s^{\prime} \cos \left(s^{\prime}-s\right) S_{12}\left(s^{\prime}, s\right) Q\left(\zeta_{L}, s^{\prime}, s\right)-\sin \left(\zeta_{L}-s\right) S_{12}\left(\zeta_{L}, s\right)\right],
$$

with $S_{12}\left(s^{\prime}, s\right)$ and $Q\left(\zeta_{L}, s^{\prime}, s\right)$ defined by Eq. (2.2) and Eq. (2.4) respectively. In this expression we define the parameter $\zeta_{L}$ as $\zeta_{L} \equiv L / t_{f}$ since we have scaled the time coordinates by the quantummechanical formation time of the parton pair,

$$
t_{f}=\frac{2 z(1-z) E}{\vec{p}^{2}}
$$

Both terms in Eq. (2.6) retain information about medium effects, although the former stems from considering the splitting inside of the medium in both amplitude and its complex-conjugate, while the second one refers to the interference between a splitting inside and another outside.

One might ask what controls the emission process. With this aim, in the following section we provide a discussion of the different time-scales which can be read off in the dipole and the factorizable piece of the quadrupole. 


\subsection{Discussion of time-scales}

Turning now to the central goal of this work, there is valuable information we can tease out from the analytical expressions of the dipole and the quadrupole. Specifically, we can draw four competing time scales appearing in Eqs. (2.2) and (2.4) after writing them in terms of the splitting variables. Two of these involve both coherence and broadening effects, and we will refer to them as the decoherence and broadening times throughout this discussion,

$$
t_{d} \sim\left(\frac{1}{\hat{q} \theta^{2}}\right)^{1 / 3}, \quad t_{b r} \sim\left(\frac{1}{\hat{q} \theta^{2} L}\right)^{1 / 2}
$$

The two remaining are the kinematical formation time, introduced in Eq. (2.7), and the already mentioned length of the medium $L$. All of them will play a striking role in the evolution of the system. On one hand, the decoherence time governs the color decoherence of the parton pair, allowing to figure out if the medium is either able to resolve the individual emitters of the pair or feel the antenna as an individual object [4]. On the other hand, the broadening time is linked to the transverse momentum broadening, which comes from the accumulation of momentum along the medium, from the formation of the antenna $t_{f}$ to the end of the medium $L$.

We have constructed the Lund plane for one splitting in Fig. 2 (left panel), which offers a clear framework to get into the discussion of the possible time orderings of the scales corresponding to the different colored areas. Let us describe the various regions sketched below:

- (A.1) $t_{f}<t_{b r}<t_{d}<L$ : Although this time-scale ordering corresponds to short formation times, particles split at large angles. Given that, the parton pair decoheres at a finite distance inside the medium, supporting the idea of in-medium vacuum-like emissions.

- (A.2) $t_{b r}<t_{f}<t_{d}<L$ : Within the green area, medium modifications are expected to show up to a large extent since the broadening time is the shortest scale controlling the evolution of the system.

- (A.3) $t_{b r}<t_{d}<t_{f}<L$ : In this region our results follow the same trend as the previous one. In particular, with the decoherence time regulating the stage before formation we appreciate a strong suppression of the formation of the dipole itself.

- (A.4) $t_{f}<L<t_{d}<t_{b r}$ : Here we support again the idea of in-medium vacuum-like emissions. The reason lies in the collinear emissions we find in this region, giving rise to color coherence across the entire medium.

- (B) $t_{f}>L$ : Medium effects are suppressed by the fact that the splitting occurs outside of the medium.

Let's summarize our results. On one hand, medium effects are expected to be visible within two different areas, more specifically on both the green and the pink ones. On the other hand, it is worth pointing out that we identify two regions of vacuum-like behaviour, namely both the red and the blue areas, supporting the notion of vacuum-like emissions inside the medium. One can check it explicitly by evaluating numerically the medium modification function into the Lund plane. As depicted in Fig. 2 (right panel), our implementation agrees well with the analytical derivation of Eq. (2.6) without overlooking the approximations taken into account. 

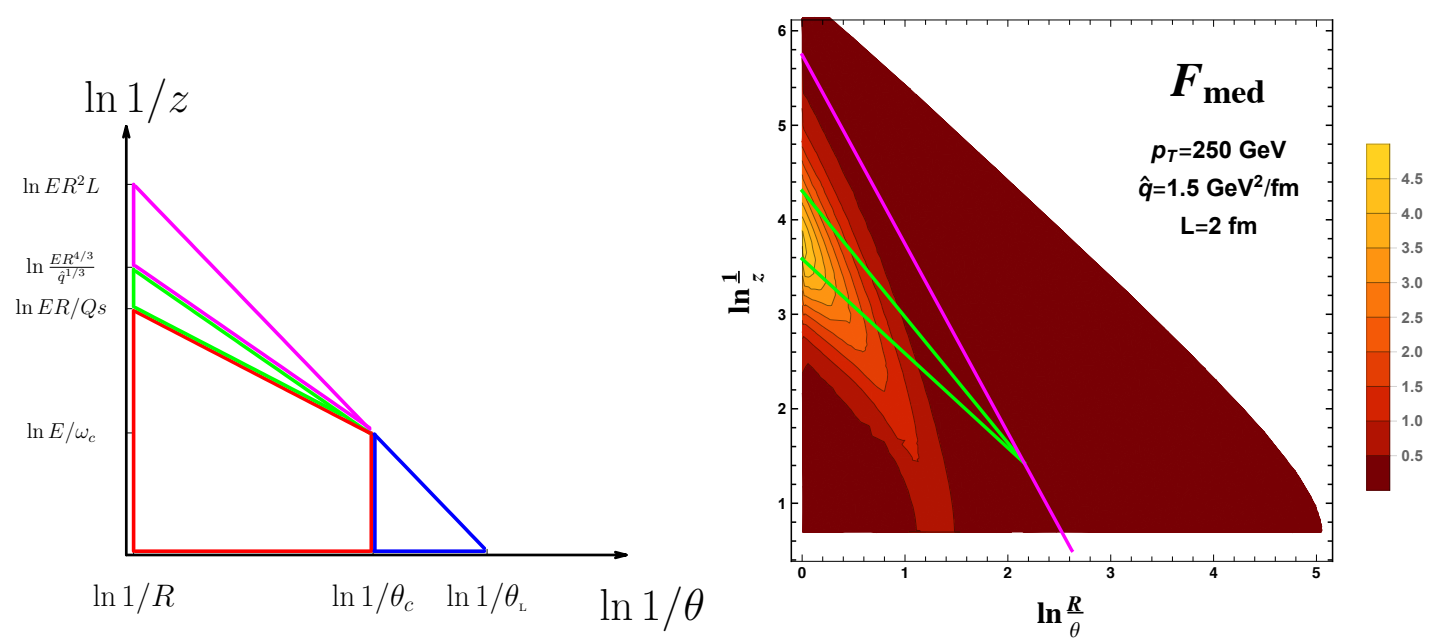

Figure 2: Depiction of the Lund plane for one vacuum splitting $\left[\theta_{c}=\left(\frac{1}{\hat{q} L^{3}}\right)^{1 / 2}, \theta_{L}=\left(\frac{1}{E L}\right)^{1 / 2}, \omega_{c}=\hat{q} L^{2}\right.$, $Q_{s}=(\hat{q} L)^{1 / 2}$ and $R$ a cone angle (left panel) and numerical evaluation of the medium modification function $F_{\text {med }}$ over the Lund plane (right panel).

\section{Discussion}

Although considering an apparently simple physical scenario, with this work we go further in understanding parton propagation in matter by keeping track the effects of taking into account the formation of the dipole itself. We point out the importance of the different time-scales appearing through the calculation which eventually seem to control the splitting process. Furthermore, we find regimes of in-medium vacuum-like emissions both analytically and numerically, which lends support to this controversial idea. Finally, it must be noted that the generalization of the process to colored splittings does not change our perception of the problem since it only alters the color algebra throughout the derivations.

Funding: This research was funded by Ministerio de Ciencia e Innovacion of Spain under project FPA2017-83814-P and Maria de Maetzu Unit of Excellence MDM-2016-0692 and by Xunta de Galicia and FEDER.

\section{References}

[1] K. Tywoniuk et al., Novel tools and observables for jet physics in heavy-ion collisions, arXiv:1808.03689.

[2] F. A. Dreyer, G. P. Salam, G. Soyez, The Lund Jet Plane, arXiv:1807.04758.

[3] J.-P. Blaizot, F. Domínguez, E. Iancu, Y. Mehtar-Tani, Medium-induced gluon branching, JHEP 01 (2013) 143, DOI:10.1007/JHEP01(2013)143, arXiv:1209.4585.

[4] Y. Mehtar-Tani, C. A. Salgado, K. Tywoniuk, The radiation pattern of a QCD antenna in a dense medium, Phys. Lett. B (2012) 707 156, arXiv:1205.5739. 